\title{
IS DAY SURGERY FOR WILSON'S OSTEOTOMY SAFE?
}

\author{
SHEO B. TIBREWAL, MARTIN V. L. FOSS
}

There is a growing need for increased use of day surgery in orthopaedics. Operations for correction of hallux valgus, such as Wilson's osteotomy have been traditionally carried out as an in-patient. We report patient compliance, results and complications of this procedure as a day case.

Patients and methods. We have reviewed 43 patients (50 feet) after Wilson's osteotomy as day cases. Their average age was 45 years (17 to 68) and the average follow-up 11.9 months ( 4 to 22 ). The medical and social implications of day surgery were explained to the patient preoperatively and leaflets were given stressing the need for support at home after the operation.

A standard Wilson's osteotomy (Wilson 1963) was performed, a plaster shoe applied and the patients were mobilised with crutches. The usual postoperative analgesia was ketoprofen suppositories. Instruction sheets were given to the patients and their general practitioners were informed. A supply of Co-dydramol was given to the patients until their further review in two to five days.

All patients were sent a questionnaire to evaluate their views on pain, analgesia required and social difficulties. Their overall satisfaction, pain relief, appearance, footwear and walking ability were recorded on percentage linear analogue scales (Downie et al 1978) and objective assessment of the foot was then recorded.

Results. All 43 patients left hospital within 10 hours of surgery: none was readmitted. The majority $(88 \%)$ reported more than $75 \%$ satisfaction after surgery and $86 \%$ were completely pain free at final follow-up. The 30 patients below the age of 55 years reported little or no inconvenience after surgery and some felt that the day surgery was less disruptive to their family life; two, however, would have preferred to stay overnight.

Most of the 13 patients above the age of 55 years felt that it was a struggle for their family and friends for the first few days after surgery. They had difficulty in making a cup of tea, going up and down stairs, etc. Three patients

S. B. Tibrewal, FRCS, Clinical Lecturer in Orthopaedic Surgery Department of Orthopaedics, Royal Victoria Infirmary, Newcastle upon Tyne NE1 4LP, England.

M. V. L. Foss, FRCS, Consultant Orthopaedic Surgeon

Luton and Dunstable Hospital, Lewsey Road, Luton, Bedfordshire LU4 0DZ, England.

Correspondence to Mr S. B. Tibrewal.

(C) 1991 British Editorial Society of Bone and Joint Surgery

0301-620X/91/2R95 \$2.00

J Bone Joint Surg [ Br] 1991 ; 73-B: 340. had to call their doctors for reassurance and further supplies of analgesic tablets. Eleven of them would have preferred admission for three days and nine would have favoured waiting six months for admission rather than coming in sooner for day-case surgery.

Most patients started to walk with a canvas boot and crutches two to seven days after surgery, many required no support, or one walking stick only within a further week or two. No serious complications were encountered. No patients required manipulation to realign the osteotomy. Two patients had minor skin-edge necrosis which healed with dressings. Two patients had oedema and pain for more than three months; two had sensitive scars; none had nonunion.

Discussion. Our study demonstrates that this operation may be performed as a day case with relative safety and a minimum of complications, provided there is adequate home help. The support of a plaster shoe, rather than a full below-knee plaster, is sufficient. The results are similar to those reported after patients have been admitted (Wilson 1963; Grace, Hughes and Klenerman 1988).

A prime consideration in selecting a patient for daycase surgery is fairly good health and an anticipated relatively short recovery period during which minimal postoperative complications can be expected. The 30minute time limit on general anaesthesia should be observed (Royal College of Surgeons of England 1985) and the operation should preferably be carried out in the morning session. Patient compliance below the age of 55 is excellent; above this age the patient should be given the option of in-patient treatment.

The authors would like to thank Mr D. Austwick, Consultant Orthopaedic Surgeon, for allowing his patients to be included in this study and Miss Susan Smith for secretarial assistance.

No benefits in any form have been received or will be received from a commercial party related directly or indirectly to the subject of this article.

\section{REFERENCES}

Downie WW, Leatham PA, Rhind VM, Pickup ME, Wright V. The visual analogue scale in the assessment of grip strength. Ann Rheum Dis 1978; 37:382-4.

Grace D, Hughes J, Klenerman L. A comparison of Wilson and Hohmann osteotomies in the treatment of hallux valgus. J Bone Joint Surg [Br] 1988; 70-B:236-41.

Royal College of Surgeons of England. Commission on the provision of surgical services. Guidelines for day case surgery. London: RCS: 1985 .

Wilson JN. Oblique displacement osteotomy for hallux valgus. $J$ Bone Joint Surg [Br] 1963; 45-B:552-6. 